\title{
Evidencia de actividad feromonal de contacto en Cerambyx welensii Küster y compuestos candidatos implicados
}

Evidence of contact sex pheromone in Cerambyx welensii Küster and identification of candidate compounds

López-Manzano, M.R. ${ }^{\text {; }}$ Sánchez-Osorio, I. ."; López-Pantoja, G. ${ }^{1}$; Domínguez, L. '; Rossell, G. ${ }^{2}$; Guerrero, Á. ${ }^{2}$

${ }^{1}$ Departamento de Ciencias Agroforestales, Escuela Técnica Superior de Ingeniería, Universidad de Huelva, 21007. Campus El Carmen (Huelva).

${ }^{2}$ Departamento de Química Biológica y Modelización Molecular, Instituto de Química Avanzada de Cataluña, CSIC, Jordi Girona 18, 08034 (Barcelona).

Teléfono: +34959217635 / Fax: +34959217560 


\title{
Resumen
}

Este trabajo analizó la existencia potencial de feromona sexual de contacto en Cerambyx welensii. Muestras de élitros y protórax (micro extracción en fase sólida) de ambos sexos se analizaron mediante CG-MS. Para confirmar la existencia de feromona de contacto en la cutícula de las hembras, se estudió la respuesta de machos de $C$. welensii frente a: hembras intactas, hembras muertas, hembras muertas sometidas a extracción hexánica de lípidos cuticulares y hembras sometidas a extracción hexánica a las que posteriormente se restituyó su propio extracto cuticular. Se encontraron 43 hidrocarburos cuticulares en élitros y 16 en protórax. Seis de ellos (11/13-Me-C27, 11-Me-C29, 2-Me-C26, 2-Me-C28, 11/12/13-Me-C27 monoeno y C27) incluyeron en conjunto, tanto en élitros como en protórax, entre el 68.1\% (élitros de machos) y el 85.4\% (protórax de hembras) del contenido lipídico total. Ninguno de los compuestos resultó específico de las hembras, pero se encontraron diferencias entre sexos en la presencia relativa para algunos compuestos. Los compuestos candidatos para actuar como feromona de contacto podrían localizarse tanto en élitros como en protórax, pudiendo actuar como componentes mayoritarios (abundancia relativa >17\% en MEFS) 11-Me-C29, C27, 2-Me-C26 y 11/13-Me-C27; y como componentes minoritarios (abundancia relativa $<2.5 \%$ ) 11/12/13-Me-C26 y C28. La mayoría de los machos $(82 \%)$ mostró la secuencia de cópula característica en Cerambycidae: Contacto antenal - Giro - Palpado - Arqueo abdominal. La retirada de componentes cuticulares redujo fuertemente el interés de los machos $(<5 \%$ de respuesta positiva tanto para el palpado como para el arqueo abdominal:); la restitución de los extractos hexánicos devolvió la respuesta positiva de los machos en un $22 \%$ de los casos, indicando que la feromona de contacto se encontraba en el extracto cuticular.

Palabras clave: 11-Me-C29, 2-Me-C26, Cerambycidae, conducta sexual, hidrocarburos cuticulares.

\begin{abstract}
The aim of this work was to determine the existence of contact sex pheromone in Cerambyx welensii (Coleoptera, Cerambycidae). Solid phase micro extraction samples from elitrum and prothorax of $C$. welensii males and females were analyzed by CG-MS. Additional bioassays were performed to confirm the existence of contact pheromone in the cuticle of $C$. welensii females. For this purpose, the response of males was checked against intact females, dead (freeze-killed) females, dead females after their cuticular waxes were removed by hexane rinsing, and females covered with their own cuticular hexane extract. A total of 43 cuticular hydrocarbons were found in elytra and 16 in prothorax. In both elytra and prothorax of insect, 6 compounds (11/13-Me-C27, 11-Me-C29, 2-Me-C26, 2-Me-C28, 11/12/13-Me-C27 monoeno y C27) comprised between $6.1 \%$ (male eliters) and $85.4 \%$ (female prothorax) of the whole hydrocarbon content. None of the compounds were specific to females, but differences between sexes were found in the relative presence of some compounds. The compounds candidate to act as a contact pheromone could be located both in elytra and prothorax, being 11-Me-C29, C27, 2Me-C26 and 11/13-Me-C27 proposed as putative components (relative abundances $>17 \%$ ), and 11/12/13-Me-C26 and C28 proposed as putative minor components (relative abundance $<2.5 \%$ ).

Most males (82\%) showed the characteristic sequence of mating-behavior stages: Antenal contact - Dashing - Licking-Abdominal bending. Males exhibited similar responses to dead females, but not to solvent-washed dead females with their cuticular hydrocarbons removed (occurrence of either licking or abdominal bending $<5 \%$ ). The mating response of males was partially restored when solvent extracts were reapplied to carcasses of solvent-washed females $(22 \%$ of positive responses), indicating that the contact pheromone was present in solvent extracts.
\end{abstract}

Keywords: 11-Me-C29, 2-Me-C26, Cerambycidae, mating behavior, cuticular hydrocarbons. 


\section{Introducción}

Las dehesas se citan como ejemplo modélico de sistema agrario de Alto Valor Natural (AVN) (WWF-Adena, 2006). El deterioro gradual del estado fisiológico que vienen sufriendo especies de Quercus en las dehesas, debido a estrés ambiental, problemas de regeneración o incidencia de agentes bióticos (patógenos de suelo y del sistema aéreo e insectos defoliadores) ha originado condiciones del arbolado propicias para el desarrollo de ciertas especies de insectos del género Cerambyx (Buse et al., 2007; Carrasco y cols., 2009; Duque-Lazo y Navarro-Cerrillo, 2017).

Dentro del género Cerambyx, una de las especies más problemáticas para el arbolado de las dehesas es Cerambyx welensii Küster (López-Pantoja et al., 2008; Sallé et al., 2014; Tiberi et al., 2016). Esta especie se ha considerado tradicionalmente como plaga secundaria (Moral, 1994), provocando la muerte de los árboles, sobre todo de pies viejos, por la creación de grandes galerías larvarias que reducen su resistencia, incrementan el riesgo de infección por patógenos (Martín et al., 2005) o de colonización secundaria por otros grandes cerambícidos, como Prinobius myardi Mulsant (Soria, 1994). El riesgo de que en situaciones de altos tamaños poblacionales $C$. welensii intensifique su capacidad de colonización de nuevos hospedantes, incluyendo pies de todas las edades y estados fisiológicos, ha sido citado con frecuencia (Moral, 1994; Navarro y Fernández, 2001). En Andalucía, la gravedad de la acción de estos insectos la reflejó el Informe de la Ponencia de Estudio Sobre la Protección del Ecosistema de la Dehesa, realizado por la Comisión de Medio Ambiente, Agricultura y Pesca del Senado (enero de 2011), reconociendo la incidencia de Cerambyx spp. como uno de los motivos de alarma justificada que pudiera poner en peligro amplias zonas adehesadas del entorno mediterráneo.

El campo de la ecología química vinculada a la entomología aplicada, y en concreto el estudio de los compuestos semioquímicos, tanto de actividad a distancia (olfativa) como de actividad por contacto, constituye uno de los pilares del control integrado de plagas forestales (Allison et al., 2004; Ginzel, 2010). Ello es debido a que la percepción de tales compuestos por los insectos se puede asociar tanto a la selección de hospedantes para la puesta y/o localización de congéneres, como a la regulación de las interacciones intraespecíficas, en particular la actividad reproductiva. Las pautas de selección de hospedantes y congéneres por cerambícidos xilófagos que afectan a frondosas, en particular $C$. welensii, siguen siendo poco conocidas. La existencia de feromonas de largo/corto alcance o de contacto ha sido constatada en más de una veintena de especies en la familia Cerambycidae (Allison et al., 2004; Pajares et al., 2010). En $C$. welensii no se ha demostrado la existencia de feromona con actividad a distancia; pero ensayos preliminares sugirieron la posible intervención de actividad feromonal de contacto durante la cópula (Sánchez-Osorio et al., 2012). Por otra parte, se ha encontrado atracción de $C$. welensii hacia trampas cebadas con sustancias que desprenden aromas propios de procesos fermentativos (Torres-Vila et al., 2012; Sánchez-Osorio et al., 2016), y también se ha encontrado asociación entre la presencia de adultos y daños de $C$. welensii y el perfil de emisión foliar de $Q$. suber, mostrando aquellos árboles con perfil de tipo limoneno 
(presencia relativa de limoneno $>30 \%$ de la emisión total de monoterpenos. Staudt et al., 2004) una mayor abundancia del insecto que pies con perfil de tipo pineno (en los cuales la emisión global de monoterpenos es dominada por $\alpha$-pineno, $\beta$-pineno y sabineno) (Sánchez-Osorio et al., 2019).

En árboles es frecuente ver a los insectos adultos deambular por troncos y ramas cuando cae la noche, siendo habitual encontrar buen número de ejemplares en torno a zonas que presentan exudados corticales (López-Pantoja et al., 2008). El apareamiento ocurre tras reconocer el macho a la hembra por sus estructuras sensoriales más notorias, las antenas (Sánchez-Osorio et al., 2012). Tras el contacto antenal se desencadena una secuencia de acciones típicas, consistentes en la precipitación hacia la hembra, palpado de su dorso y arqueo abdominal (Sánchez-Osorio et al., 2012). El palpado es una pauta llamativa, consistente en unos movimientos laterales de la cabeza del macho, a modo de vaivén, fundamentalmente sobre el pronoto e inicio de los élitros de la hembra, rozando su superficie con los palpos bucales; simultáneamente a este acción suele tener lugar el arqueo abdominal que propicia la cópula.

La cutícula de los insectos cumple funciones como la protección de órganos internos, anclaje para los músculos actuando como exoesqueleto, protección frente a factores bióticos y abióticos (acción de patógenos, desecación, humedad excesiva) o evitar la entrada de solventes (de la Cruz, 2006). Muchos lípidos que se encuentran en la cutícula de los insectos actúan como feromonas, resultando informativas sobre su edad, sexo y estado reproductivo (Chiang et al., 2016). Dichas feromonas de contacto, compuestas por hidrocarburos cuticulares, provocan también el comportamiento de cópula de los machos en Cerambycidae (Rodríguez, 2014; Ginzel et al., 2016). Estos hidrocarburos cuticulares pueden representar mezclas complejas: hidrocarburos de cadena lineal (n-alcanos), hidrocarburos insaturados (olefinas) y ramificados con uno o varios metilos (metilalcanos) en posiciones terminales o intermedias de la cadena (Lockey, 1985).

Este estudio pretende contribuir al conocimiento de las pautas de reconocimiento sexual para la cópula en $C$. welensii, en particular la determinación de compuestos candidatos a actuar como feromona sexual de contacto incitadora de la cópula. Los objetivos que se han planteado son:

1. Analizar los componentes cuticulares de machos y hembras en $C$. welensii para detectar diferencias entre sexos que puedan sugerir la presencia de compuestos estimuladores de la cópula (feromona sexual de contacto).

2. Completar estudios preliminares previos mediante bioensayos (Sánchez-Osorio et al., 2012) para comprobar la existencia de feromona de contacto cuticular estimuladora de la cópula. 


\section{Material y Métodos}

\subsection{Análisis cromatográfico de hidrocarburos cuticulares}

Ejemplares adultos inmaduros fueron recolectados en campo durante su período de letargo (octubre de 2012 en la finca "Las Espechillas", TM de Huévar, Sevi1la), y mantenidos individualmente en tubos de PVC tapados, dentro de urnas de metacrilato $(50 \times 40 \times 20 \mathrm{~cm} ; 10$ insectos por urna). Estos ejemplares inmaduros suelen permanecer en su cámara pupal entre septiembre y mayo, antes de iniciar el período de vuelo; su abdomen presenta una tonalidad amarillenta característica. Cuando los insectos iniciaron su actividad, saliendo de los tubos de PVC (comienzos de junio de 2013) se proveyó a las urnas de agua azucarada y pedazos de corcho, permitiendo que los insectos interaccionasen de modo similar a como lo harían en el campo (movimientos, enfrentamientos, intentos de cópula, oviposición, etc.). El muestreo de hidrocarburos cuticulares de los insectos se realizó a partir de microextracción en fase sólida (MEFS), a finales de junio de 2013. Para ello se frotó el filamento adsorbente (sílice recubierta con polidimetilsiloxano, de 100 micras de espesor y $1 \mathrm{~cm}$ de largo. Sigma-Aldrich, Madrid. En adelante "fibra") en la cutícula de los élitros y protórax de los insectos, del modo siguiente (Fig. 1):

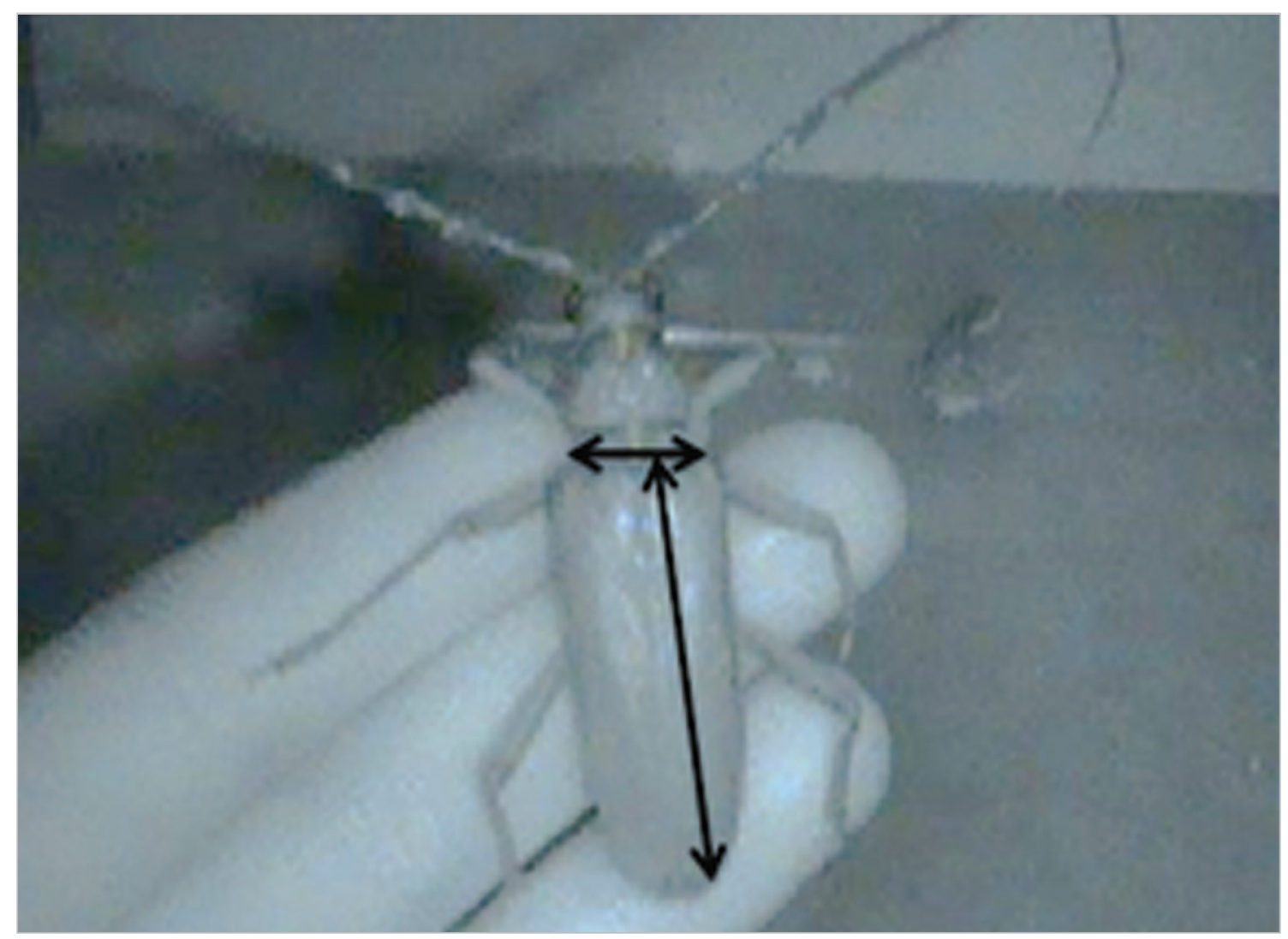

Figura 1. Direcciones de frotado del filamento para el muestreo, mediante microextracción en fase sólida (MEFS), de hidrocarburos cuticulares presentes en pronoto y élitros de Cerambyx welensii. 
* Élitros: la fibra se orientó oblicuamente (unos $60^{\circ}$ ) respecto al eje longitudinal de los élitros. Se realizaron tres frotaciones de la fibra a lo largo del élitro derecho. En pruebas preliminares se observó que una mayor intensidad de frotado aumentaba innecesariamente la cantidad de muestra recolectada, lo cual dificultaba la separación cromatográfica.

* Protórax: frotado dos veces de la fibra sobre la parte distal, no rugosa, del pronoto, en dirección perpendicular al eje longitudinal del insecto.

Se obtuvieron muestras MEFS de élitros a partir de 9 ejemplares de cada sexo. En otros 10 ejemplares diferentes ( 5 de cada sexo) se obtuvieron muestras MEFS de protórax. Antes de cada muestreo, la fibra fue acondicionada a $250{ }^{\circ} \mathrm{C}$ durante al menos una hora, siguiendo las especificaciones del fabricante. Inmediatamente después de la toma de muestras de cada insecto, la fibra se insertó en el inyector del cromatógrafo (CG-MS Thermo Finnigan 2000. Columna Thermo TR-5MS, $30 \mathrm{~m} \mathrm{x}$ $0.25 \mathrm{~mm}$ ID x 0.25 micras). Las condiciones de análisis fueron: rango de escaneo de $34-570 \mathrm{~m} / \mathrm{z}$, con frecuencia de una muestra por segundo; temperatura del inyector, $250^{\circ}$.

\subsection{Bioensayo}

Los insectos empleados en los bioensayos se recolectaron en las provincias de Huelva (finca "Los Centenales", TM de Gibraleón) y Sevilla (finca "Las Espechillas", TM de Huévar) entre los meses de mayo y junio de 2018. Los ejemplares se mantuvieron a temperatura e iluminación del laboratorio, en urnas similares a las descritas anteriormente y en las mismas condiciones (machos y hembras por separado; 7 ejemplares por urna). Cada macho se identificó mediante un pequeño número en el ápice de uno de los élitros.

Para comprobar la hipótesis de que en el reconocimiento para la cópula intervienen feromonas de contacto, se empleó el bioensayo propuesto por Ginzel et al. (2003a), según el cual se observó la reacción de machos cuando se enfrentaban sucesivamente a hembras en distintas condiciones, a partir de la siguiente secuencia:

\section{Hembra viva.}

2. Hembra muerta (la hembra anterior, tras un período de congelación de una hora, y posterior aclimatación a temperatura ambiente).

3. La misma hembra muerta, tras la extracción de sus lípidos cuticulares (en adelante, "Hembra Extraída"). La extracción se realizó mediante doble inmersión del insecto en $10 \mathrm{ml}$ de hexano durante 3 minutos. El extracto resultante para cada hembra $(20 \mathrm{ml})$ se concentró en flujo de nitrógeno hasta alcanzar $1.2 \mathrm{ml}$, lo que se consideró como un "Equivalente de Hembra" (EH).

4. El mismo ejemplar, al cual se le aplicó su propio extracto hexánico (en ade- 
lante, "Hembra Restituida"). La dosis total aplicada a cada hembra fue de 1 EH (1.2 ml), lo que se alcanzó tras la aplicación sucesiva de tres alícuotas de $0.4 \mathrm{ml}$ cada una (denominadas Restitución 1/3 EH, Restitución 2/3 EH y Restitución $1 \mathrm{EH}$, respectivamente). El comportamiento de los machos se anotó después de cada una de las restituciones.

Tras pruebas preliminares se descartó la observación por parejas de insectos debido a la excesiva pasividad que tienden a mostrar. Esta pasividad se aminoró considerablemente cuando las hembras se presentaron a grupos de machos. Por tanto, las observaciones durante los bioensayos se realizaron empleando una hembra para cada grupo de machos mantenido en cada urna.

Los bioensayos se realizaron con un total de 5 hembras de similares dimensiones (longitud de élitros $3.24 \pm 0.37 \mathrm{~cm}$; anchura de élitros en su base $1.24 \pm 0.15$ $\mathrm{cm}$ ), introduciendo una hembra distinta en cada urna de machos (en total, $35 \mathrm{em}$ parejamientos distintos observados). A medida que cada macho interaccionaba con la hembra y se anotaba su comportamiento, era cuidadosamente retirado a una segunda urna de las mismas características que la de partida. Las observaciones se realizaron en horario crepuscular y nocturno, cuando los insectos comenzaron su actividad y mientras se mostraban activos (generalmente entre las 20:00 y las 02:00 horas). Para la observación se empleó una linterna con luz roja de intensidad regulable. Se intentó realizar la menor manipulación de machos y hembras, lo cual se conseguía propiciando que los insectos entraran a placas Petri de plástico de $10 \mathrm{~cm}$ de diámetro; cuando fue necesario, se usaron guantes. El tiempo de observación para cada grupo de machos fue de 20 minutos. En general, se consideró como respuesta positiva de los machos cuando los insectos realizaron alguna de las acciones siguientes: Giro tras el contacto antenal $(C)$ seguido de detención del movimiento del macho y mantenimiento en contacto con la hembra, alineamiento del cuerpo del macho con la hembra e inicio de palpado $(P)$, o arqueo abdominal $(A)$ (Lacey et al., 2008; Silk et al., 2011). En cualquier caso, cuando algún macho continuó su marcha tras el contacto antenal y sin realizar ninguna de las otras acciones descritas, se consideró respuesta negativa $(I)$. Los bioensayos se grabaron con videocámara.

\subsection{Análisis estadístico}

En el estudio de los lípidos cuticulares, el valor de análisis para cada compuesto fue su área relativa (\%) respecto a la suma de las áreas de todos los compuestos detectados en cada insecto. La comparación de áreas medias entre sexos se realizó a partir del test robusto de Yuen-Welch, a partir de medias recortadas al $20 \%\left(\mathrm{Me}_{0.2}\right.$, media aritmética obtenida después de eliminar el $20 \%$ de los valores más altos y el $20 \%$ de los valores más bajos en cada muestra de partida).

Para analizar el resultado del bioensayo, se anotó el porcentaje de insectos que mostró respuesta positiva, además del tipo de acción exhibida $(C, P, A$ o $I)$. La comparación entre los porcentajes de respuesta para cada una de las acciones entre los distintos tratamientos se realizó mediante el test $Q$, de Cochran, realizando las com- 
paraciones múltiples mediante el test de McNemar (método de permutaciones, ajuste mediante el método de false discovery rate). Los análisis estadísticos se realizaron con el programa $R$, versión 3.1.0, utilizando $\alpha=0.05$ como nivel de significación.

\section{Resultados}

\subsection{Análisis cromatográfico de hidrocarburos cuticulares}

El estudio de los hidrocarburos que integran el contenido lipídico cuticular, mediante micro extracción en fase sólida, determinó la presencia de un total de 43 compuestos en élitros y 16 en protórax (Tab. 1). Tanto en élitros como en protórax, seis compuestos (11/13-Me-C27, 11-Me-C29, 2-Me-C26, 2-Me-C28, 11/12/13-Me$\mathrm{C} 27$ monoeno y $\mathrm{C} 27$ ) tuvieron presencia relativa $>5 \%$ y comprendieron, en conjunto, entre el $68.1 \%$ (élitros de machos) y el $85.4 \%$ (protórax de hembras) del contenido lipídico total detectado (Tab. 1). Treinta compuestos presentaron en élitros una abundancia relativa $<1 \%$ en hembras (26 compuestos en machos) (Tab. 1). Cinco compuestos tuvieron en protórax una presencia $<1 \%$ en hembras ( 1 en machos) (Tab. 1). El compuesto con mayor presencia relativa tanto en élitros como en protó$\operatorname{rax}(11 / 13-\mathrm{Me}-\mathrm{C} 27)$ se presentó en similares cuantías en machos y hembras, pero en mayor valor relativo en las hembras (Test de Yuen-Welch, $P=0.09$ ).

No se encontró ningún componente cuticular específico de las hembras, pero sí se detectó una proporción significativamente diferente entre sexos para determinados compuestos (12 compuestos en élitros, 6 en protórax). De entre ellos, presentaron tasas significativamente mayores en élitros de hembras los compuestos 11-MeC29 y No identificado 1; en el protórax resultaron ser los compuestos 11-Me-C29 y No identificado 5.

Tanto en élitros como en protórax, 11-Me-C29 fue el compuesto con mayor presencia en hembras de entre los que superaron significativamente su presencia en machos; por sí solo comprendió en hembras el 17.6\% del contenido lipídico en élitros y el $21.8 \%$ en protórax. Los otros dos compuestos con presencia significativamente mayor en hembras (ambos no identificados) tuvieron un carácter de componentes minoritarios (presencia $<1 \%$ tanto en élitros como en protórax).

Diez compuestos identificados en los élitros tuvieron en los machos presencia relativa significativamente mayor a la encontrada en hembras (Tab. 1), de ellos sólo el 2-Me-C26 tuvo una presencia reseñable (8.86\% como media para ambos sexos), los demás tuvieron una presencia relativa $<3.5 \%$. En protórax, fueron cinco los compuestos cuya presencia relativa en machos superó significativamente a la hallada en hembras; de ellos de nuevo el 2-Me-C26 (7.73\% como media de ambos sexos) junto al C27 (5.72\% y $10.43 \%$, hembras y machos respectivamente) tuvieron presencia destacable, los demás tuvieron relevancia inferior al $2.7 \%$. 
Tabla 1. Presencia relativa (\%) de los compuestos detectados mediante microextracción en fase sólida (MEFS) (Me0.2 \pm EE0.2) en élitros y protórax de Cerambyx welensii. En negrita se resaltan los compuestos con presencia $>5 \%$, así como los valores significativamente diferentes entre sexos para cada parte muestreada (Test de Yuen-Welch). TR, tiempo de retención (minutos).

\begin{tabular}{|c|c|c|c|c|c|c|}
\hline \multirow[b]{2}{*}{ Compuestos } & \multirow[b]{2}{*}{ TR } & \multicolumn{2}{|c|}{ Élitros } & \multicolumn{2}{|c|}{ Protórax } & \multirow[b]{2}{*}{ Iones característicos } \\
\hline & & Я & $\pi$ & ? & $\pi$ & \\
\hline $\mathrm{C} 18$ & 30.24 & $0.02 \pm 0.01$ & $0.03 \pm 0.01$ & - & - & $268(\mathrm{M}+)$ \\
\hline 9-Me-C19 & 32.81 & $0.02 \pm 0.01$ & $0.01 \pm 0$ & - & - & $\begin{array}{c}140 / 141 ; 168 / 169 \\
282(\mathrm{M}+)\end{array}$ \\
\hline $\mathrm{C} 20$ & 33.72 & $0.02 \pm 0.01$ & $0.02 \pm 0.01$ & - & - & $282(\mathrm{M}+)$ \\
\hline $10-\mathrm{Me}-\mathrm{C} 20$ & 34.21 & $0.01 \pm 0.01$ & $0.02 \pm 0.01$ & - & - & $\begin{array}{c}\text { 154/155;168/169; } \\
296(\mathrm{M}+)\end{array}$ \\
\hline 2-Me-C20 & 34.52 & $0.02 \pm 0.01$ & $0.03 \pm 0.01$ & - & - & $253 / 254 ; 296(\mathrm{M}+)$ \\
\hline $\mathrm{C} 21$ & 35 & $0.05 \pm 0.02$ & $0.12 \pm 0.03$ & - & - & $296(\mathrm{M}+)$ \\
\hline 11-Me-C21 & 35.4 & $0.11 \pm 0.01$ & $0.25 \pm 0.15$ & - & - & $168 / 169 ; 310(\mathrm{M}+)$ \\
\hline C22eno & 35.8 & $0.02 \pm 0.01$ & $0.03 \pm 0$ & - & - & $308(\mathrm{M}+)$ \\
\hline $\mathrm{C} 23$ & 37.1 & $0.06 \pm 0.02$ & $0.09 \pm 0.02$ & - & - & $324(\mathrm{M}+)$ \\
\hline 11-Me-C23 & 37.44 & $0.1 \pm 0.02$ & $0.12 \pm 0.02$ & - & - & $\begin{array}{c}\text { 168/169;196/197; } \\
338(\mathrm{M}+)\end{array}$ \\
\hline No identificado 1 & 38.02 & $0.8 \pm 0.13$ & $0.18 \pm 0.04$ & - & - & - \\
\hline 12-Me-C24 & 38.31 & $0.05 \pm 0.01$ & $0.07 \pm 0.02$ & - & - & $182 / 183 ; 352(\mathrm{M}+)$ \\
\hline 2-Me-C24 & 38.59 & $0.07 \pm 0.02$ & $0.13 \pm 0.03$ & - & - & $352(\mathrm{M}+)$ \\
\hline $\mathrm{C} 25$ & 38.9 & $0.31 \pm 0.03$ & $0.47 \pm 0.07$ & - & - & $352(\mathrm{M}+)$ \\
\hline 11/13-Me-C25 & 39.17 & $0.8 \pm 0.11$ & $1.02 \pm 0.11$ & $0.51 \pm 0.10$ & $1.16 \pm 0.16$ & $\begin{array}{c}168 / 169 ; 224 / 225 \\
366(\mathrm{M}+)\end{array}$ \\
\hline 2-Me-C25 & 39.38 & $0.15 \pm 0.06$ & $0.37 \pm 0.04$ & - & - & $323 / 324 ; 366(\mathrm{M}+)$ \\
\hline $\mathrm{C} 26$ & 39.71 & $0.82 \pm 0.04$ & $1.06 \pm 0.07$ & $0.47 \pm 0.13$ & $1.02 \pm 0.05$ & $366(\mathrm{M}+)$ \\
\hline 11/12/13-Me-C26 & 39.94 & $1.23 \pm 0.11$ & $1.54 \pm 0.05$ & $0.9 \pm 0.13$ & $1.41 \pm 0.10$ & $\begin{array}{l}\text { 168/169;182/183; } \\
\text { 196/197;224/225; } \\
\text { 238/239;380(M+) }\end{array}$ \\
\hline 2-Me-C26 & 40.19 & $8.48 \pm 0.4$ & $9.25 \pm 0.53$ & $6.5 \pm 0.52$ & $8.96 \pm 0.12$ & $337 / 338 ; 380(\mathrm{M}+)$ \\
\hline C27eno & 40.31 & $0.31 \pm 0.03$ & $0.63 \pm 0.11$ & - & - & 97/98;111/112;378 (M+) \\
\hline $\mathrm{C} 27$ & 40.48 & $3.18 \pm 2.04$ & $5.53 \pm 2.59$ & $5.72 \pm 1.47$ & $10.43 \pm 0.89$ & $380(\mathrm{M}+)$ \\
\hline $\begin{array}{c}\text { 11/12/13-Me-C27 } \\
\text { monoeno }\end{array}$ & 40.66 & $5.72 \pm 1.16$ & $6.91 \pm 0.68$ & $7.59 \pm 1.59$ & $4.63 \pm 0.68$ & $\begin{array}{c}\text { 97/98;111/112;168/169; } \\
\text { 182/183;196/197;224 } \\
\text { /225;238/239; } \\
252 / 253 ; 392(\mathrm{M}+)\end{array}$ \\
\hline 11/13-Me-C27 & 40.71 & $32.95 \pm 3.83$ & $26.3 \pm 2.37$ & $37.36 \pm 6.68$ & $37.59 \pm 1.24$ & $\begin{array}{c}168 / 169 ; 196 / 197 ; 224 / \\
225 ; 252 / 253 ; 394(\mathrm{M}+)\end{array}$ \\
\hline 11,13-Dime-C27 & 40.88 & $1.18 \pm 0.17$ & $1.47 \pm 0.07$ & $1.31 \pm 0.22$ & $1.56 \pm 0.40$ & $\begin{array}{c}168 / 169 ; 196 / 197 ; 239 / \\
240 ; 267 / 268 ; 408(\mathrm{M}+)\end{array}$ \\
\hline
\end{tabular}


Viene de pág. anterior

\begin{tabular}{|c|c|c|c|c|c|c|}
\hline \multirow[b]{2}{*}{ Compuestos } & \multirow[b]{2}{*}{ TR } & \multicolumn{2}{|c|}{ Élitros } & \multicolumn{2}{|c|}{ Protórax } & \multirow[b]{2}{*}{ Iones característicos } \\
\hline & & 9 & ô & 운 & 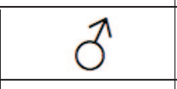 & \\
\hline 3-Me-C27 & 41.05 & $0.79 \pm 0.04$ & $0.82 \pm 0.08$ & - & - & 364/365;394 (M+) \\
\hline $\mathrm{C} 28$ & 41.21 & $1.88 \pm 0.12$ & $2.24 \pm 0.10$ & $1.33 \pm 0.29$ & $2.64 \pm 0.10$ & $394(\mathrm{M}+)$ \\
\hline $\begin{array}{l}\text { 11/12/13/14- } \\
\text { Me-C28 }\end{array}$ & 41.41 & $2.92 \pm 0.16$ & $2.13 \pm 0.53$ & $2.38 \pm 0.17$ & $2.58 \pm 0.22$ & $\begin{array}{c}\text { 168/169;182/183; } \\
\text { 196/197;210/21; } \\
224 / 225 ; 238 / 239 ; 252 / \\
253 ; 266 / 267 ; 408(\mathrm{M}+)\end{array}$ \\
\hline No identificado 2 & 41.55 & $0.28 \pm 0.12$ & $0.15 \pm 0.03$ & - & - & - \\
\hline No identificado 3 & 41.59 & $0.22 \pm 0.01$ & $0.29 \pm 0.09$ & - & - & - \\
\hline 2-Me-C28 & 41.64 & $6.39 \pm 0.54$ & $6.16 \pm 0.27$ & $5.09 \pm 1.46$ & $6.91 \pm 0.15$ & 365/366;408 (M+) \\
\hline $\mathrm{C} 29$ & 41.9 & $2.42 \pm 0.10$ & $3.11 \pm 0.21$ & $2.21 \pm 0.64$ & $3.04 \pm 0.17$ & $408(\mathrm{M}+)$ \\
\hline 11-Me-C29 & 42.12 & $17.63 \pm 0.73$ & $14.13 \pm 1.11$ & $21.18 \pm 0.70$ & $13.53 \pm 0.82$ & $\begin{array}{c}\text { 168/169;280/281; } \\
422(\mathrm{M}+)\end{array}$ \\
\hline 11,15-Dime-C29 & 42.28 & $1.71 \pm 0.18$ & $1.56 \pm 0.12$ & $1.79 \pm 0.40$ & $1.37 \pm 0.11$ & $\begin{array}{c}168 / 169 ; 224 / 225 ; 239 / \\
240 ; 295 / 296 ; 436(\mathrm{M}+)\end{array}$ \\
\hline No identificado 5 & 42.43 & $0.66 \pm 0.15$ & $0.83 \pm 0.17$ & - & - & 168/169;422 (M+) \\
\hline $\mathrm{C} 30$ & 42.63 & $0.43 \pm 0.10$ & $0.97 \pm 0.06$ & - & - & $422(\mathrm{M}+)$ \\
\hline 12/14/16-Me-C30 & 42.84 & $0.47 \pm 0.14$ & $0.41 \pm 0.10$ & - & - & $\begin{array}{c}182 / 183 ; 210 / 211 ; 224 / \\
225 ; 238 / 239 ; 252 / 253 \\
280 / 281 ; 436(\mathrm{M}+)\end{array}$ \\
\hline 2-Me-C30 & 43.12 & $1 \pm 0.10$ & $1.67 \pm 0.11$ & $0.84 \pm 0.21$ & $1.53 \pm 0.29$ & $\begin{array}{c}\text { 393/394;421/422; } \\
436(\mathrm{M}+)\end{array}$ \\
\hline $\mathrm{C} 31$ & 43.45 & $0.68 \pm 0.05$ & $1.43 \pm 0.23$ & - & - & $436(\mathrm{M}+)$ \\
\hline $\begin{array}{l}\text { 15-Me-C31 } \\
\text { monoeno }\end{array}$ & 43.69 & $1.53 \pm 0.40$ & $2.21 \pm 0.12$ & $1.6 \pm 0.30$ & $1.57 \pm 0.14$ & $\begin{array}{c}\text { 97/98;111/112; } \\
\text { 224/225;252/253; } \\
\quad 448(\mathrm{M}+)\end{array}$ \\
\hline $\begin{array}{l}\text { Coeluye 11- } \\
\text { Me-C31 } \\
\text { con monoeno }\end{array}$ & 44.26 & $0.26 \pm 0.04$ & $0.21 \pm 0.04$ & - & - & $\begin{array}{c}97 / 98 ; 111 / 112 ; 168 / 169 ; \\
450(\mathrm{M}+)\end{array}$ \\
\hline 11-Me-C31 & 44.72 & $0.38 \pm 0.12$ & $1.45 \pm 1.02$ & - & - & $168 / 169 ; 450(\mathrm{M}+)$ \\
\hline No identificado 4 & 45.01 & $0.33 \pm 0.08$ & $0.31 \pm 0.05$ & - & - & - \\
\hline No identificado 5 & 45.93 & $1.74 \pm 0.36$ & $1.44 \pm 0.56$ & $0.67 \pm 0.05$ & $0.04 \pm 0.02$ & - \\
\hline
\end{tabular}

\subsection{Bioensayo}

Los machos mostraron un comportamiento característico de cara a la cópula, exhibiendo la secuencia general Contacto antenal $(C)$ - Palpado $(P)$ - Arqueo abdominal $(A)$ - Cópula. Un $82 \%$ (promedio entre las actitudes $C, P$ y $A$ ) de los machos exhibió esta secuencia. Las hembras, en cambio, se mostraron en general pasivas en este proceso. Un gran porcentaje de los machos $(67 \%$, promedio de $C, P$ y $A$ ) siguió 
esta secuencia también frente a hembras muertas $(1 \leq Z \leq 2 ; 0.08, \leq P \leq 0.33$; según la acción $C, P$ o $A$ considerada). La respuesta positiva de los machos frente a hembras muertas a las que se retiró el contenido lipídico cuticular (Hembra Extraída) fue irrelevante (5\% como promedio de las observaciones de $C, P$ o $A)(4.28 \leq Z \leq 4.71$, $P<0.001$; para los contrastes Hembra Muerta vs Hembra Extraída, en cada acción observada) (Fig. 2).

La evolución de dos de las acciones $(C$ y $P$ ) fue similar en los tratamientos en los cuales se restituyó el extracto hexánico cuticular a los cuerpos de cada Hembra Extraída (Fig. 2). Así, mientras que el tratamiento Restitución 1/3 EH no originó mejora significativa en la respuesta positiva de los machos, tanto $C$ como $P$ aumentaron en la Restitución 2/3 EH (observándose $C$ en el 29\% de los machos, y $P$ en el 24\%); la misma respuesta se mantuvo tras Restitución 1 EH (Test McNemar: $Z \leq$ 2.33, $0.05<P \leq 0.03$; para las acciones $C$ y $P$ y los contrastes Restitución $2 / 3$ EH vs Hembra Extraída). La acción de arqueo abdominal siguió, en cambio, una evolución diferente a $C$ y $P$, y no recuperó significativamente sus tasas de ocurrencia tras la restitución de extractos hexánicos (Test McNemar: $Z=0.58, P=0.651$; para Restitución 1 EH vs Hembra Extraída) (Fig. 2).

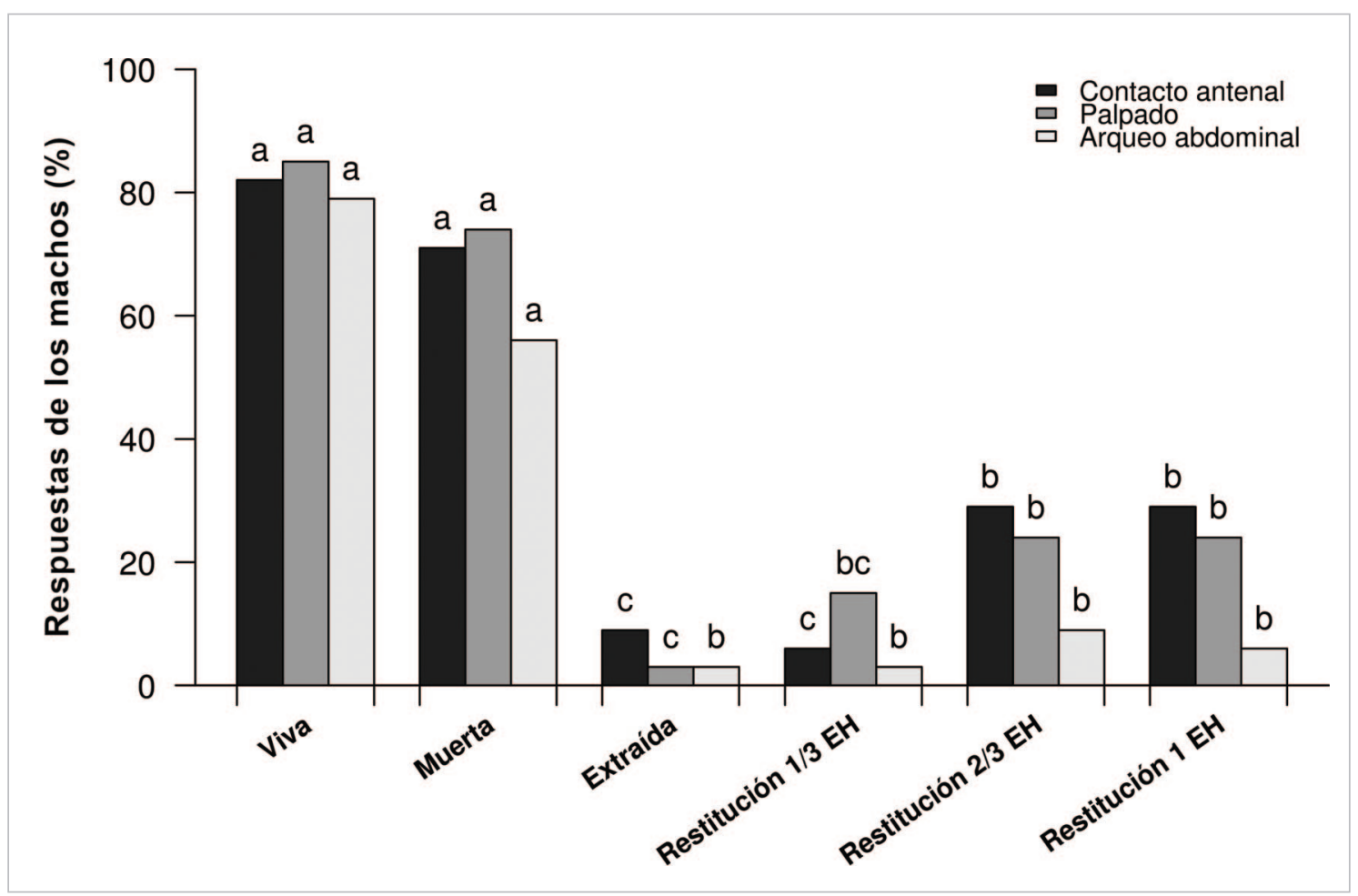

Figura 2. Respuestas de los machos de Cerambyx welensii (\%) durante el protocolo de apareamiento, mostradas ante diferentes estados de presentación de las hembras: hembras vivas, hembras muertas, hembras muertas a las cuales se extrajo el contenido lipídico cuticular, y las mismas hembras muertas a las que se restituyó su extracto cuticular $(\mathrm{EH})$ aplicado en tres dosis sucesivas. Para cada acción indicadora de respuesta de los machos (contacto antenal, palpado, arqueo abdominal), letras distintas muestran diferencias significativas según el estado de la hembra presentada (Test $Q$ de Cochran seguido de contrastes múltiples según el test de McNemar). 


\section{Discusión}

En un estudio preliminar el comportamiento de machos de $C$. welensii frente a hembras muertas a las que, tras serle retirada la cubierta lipídica cuticular, se les había restituido su propio extracto hexánico cuticular, sugirió la existencia de feromona de contacto en esta especie (Sánchez-Osorio et al., 2012). Entre los compuestos de actividad feromonal por contacto que se han encontrado en Cerambycidae, se han citado hidrocarburos de 25 a 28 átomos de carbono (Ginzel, 2010). Como ejemplo, nC25 se ha identificado como compuesto con actividad feromonal de contacto en Xylotrechus colonus F. (Ginzel et al., 2003a), 7-Me-C27 se ha citado para Neoclytus acuminatus F. (Lacey et al., 2008), y 11-Me-C27 para Tetropium fuscum F. (Silk et al., 2011).

Las pautas de comportamiento que mostraron en este estudio los machos de $C$. welensii de cara a la cópula sugieren la participación de estimulación química por contacto (feromona de contacto femenina). Esta estimulación podría estar determinada por una combinación de hidrocarburos cuticulares que se encuentran en diferente proporción en ambos sexos tanto en élitros como en protórax. Entre los compuestos candidatos figurarían 11-Me-C29 (en especial) junto con C27 y 2-Me-C26. El compuesto 11/13-Me-C27 también merecería consideración por ser el dominante en ambos sexos y, aunque con menor claridad que los otros compuestos, por su mayor presencia en la composición cuticular de las hembras, lo que podría llegar a otorgarle un papel relevante. Un segundo grupo de compuestos podrían actuar como componentes minoritarios: 11/12/13-Me-C26 y C28 (élitros y protórax); C26, C29, 2-Me-C30 y C31 (en élitros); y 11/13-Me-C25 (en protórax).

La participación como feromona de contacto de compuestos presentes en ambos sexos, pero con mayor abundancia en hembras que en machos, ha sido ya citada en Cerambycidae. Esto ocurre por ejemplo con 11-Metil-heptacosano (11-Me-C27), que se ha citado como componente de la feromona de contacto de T. fuscum y Tetropium cinnamopterum (Kirby) (Silk et al., 2011). En Monochamus galloprovincialis (Olivier), la falta de compuestos cuticulares claramente distintivos de las hembras, unido a que la mayoría de los compuestos encontrados tuvieron una presencia relativa muy baja, sugirió que los compuestos con actividad feromonal podrían estar presentes en pequeñas cantidades, atribuyéndose la distinción sexual a la diferente proporción en que los componentes cuticulares aparecían en cada sexo (Ibeas et al., 2008). En cambio, la cutícula de hembras de Megacyllene robiniae (Forster) presentó mucho más $(Z)$-9-pentacoseno que la de machos (40 y $0.3 \%$ del contenido total, respectivamente), mientras que en Xylotrechus colonus F., 11/13/15-MeC29 resultó más abundante en machos que en hembras (Ginzel et al., 2003b).

En este contexto ya se ha atribuido en la familia Cerambycidae un papel muy importante a las antenas, facilitando el encuentro entre ambos sexos y el apareamiento, al permitir el reconocimiento de feromonas de contacto (Ginzel et al., 2003a; Lopes et al., 2005). Pero en la percepción de este tipo de compuestos por C.welensii podrían tener una fuerte relevancia los palpos bucales (maxilares y/o labiales). Así, Sánchez-Osorio et al. (2012) encontraron que, si bien la privación de la sensibilidad antenal en los machos de $C$. welensii suprimió la actitud de giro hacia las hembras, permitir el contacto con los palpos (incluso con las antenas recubiertas de 
parafilm) aumentó el porcentaje de intentos de cópula hasta el 80\%. Este comportamiento parece sugerir, adicionalmente, una baja relevancia de la estimulación visual en la localización de la pareja cuando no actúa el contacto antenal, como se ha propuesto para Nadezhdiella cantori (Hope) (Wang et al., 2002), lo que podría ser algo generalizado en cerambícidos sin dimorfismo cromático pero que presentan feromona sexual de corto alcance o de contacto (Wang, 2002).

Los resultados obtenidos en nuestro bioensayo muestran que $C$. welensii realiza un comportamiento específico durante el apareamiento, por medio de la secuencia Contacto antenal - Palpado - Arqueo abdominal - Cópula. Dicho comportamiento es semejante al citado para otras especies de Cerambycidae (Wang et al., 2002; Barbour et al., 2007, Ibeas et al., 2008). En nuestro estudio, los machos reaccionaron positivamente en general frente a hembras muertas. La extracción de componentes cuticulares a las hembras muertas disminuyó fuertemente el interés de los machos (respuesta positiva en torno al 5\% de los casos), pero la restitución de los extractos hexánicos cuticulares a esos mismos ejemplares de hembras devolvió parcialmente el interés de los machos (respuesta positiva en hasta un $29 \%$ de los machos, evidenciada mediante la detección de la marcha tras el contacto antenal $-C-$ ). Los resultados encontrados en el presente estudio son semejantes a otros citados en Cerambycidae (Ginzel et al., 2003a, Ibeas et al., 2008, Sacristán et al., 2017) donde los machos intentaron aparearse con hembras vivas o muertas, las rechazaron tras la inmersión de las hembras en hexano y volvieron a recuperar el interés cuando las hembras extraídas eran cubiertas con su extracto hexánico. Los porcentajes de respuesta positiva de los machos encontradas en otros estudios, ante hembras extraídas a las cuales se les restituyó su extracto cuticular, son variables, desde el 100\% en Monochamus sutor L. y M. galloprovincialis (Sacristán et al., 2017) a un 39\% de respuesta en Megacyllene caryae (Gahan) para dosis de extractos de 0.6 a $0.7 \mathrm{EH}$ (Ginzel et al., 2003a). En nuestro estudio, la respuesta positiva media (a partir de las tres acciones: $C, L$ y $A$ ) ante hembras extraídas con restitución de extractos a dosis de $2 / 3 \mathrm{EH}$ fue del $22 \%$. Es interesante resaltar que el recubrimiento con extractos de hembras puede no devolver el interés sexual a los machos. Esto se observó en $T$. fuscum y T. cinnamopterum, incluso cuando bioensayos con compuestos cuticulares individualizados habían demostrado la presencia de feromona sexual de contacto (Silk et al., 2011).

Silk et al. (2011) encontraron que un 10\% de los machos de T. fuscum y T. cinnamopterum exhibía aún respuesta sexual ante hembras muertas a las que se extrajeron los lípidos cuticulares mediante inmersión en hexano, concluyendo que la eficacia de este procedimiento a la hora de desproveer de componentes cuticulares podría depender del tiempo de extracción. En nuestro estudio, la proporción de respuesta positiva ante hembras extraídas $(<10 \%$ para la acción $C,<5 \%$ para $L$ y $6 \%$ para $A$ ) podría haberse debido a una retirada incompleta de los compuestos cuticulares con eventual actividad feromonal, pero también podría ser motivada por una estimulación mecánica a partir de la silueta y rugosidad del cuerpo de las hembras. Los estímulos táctiles podrían tener un papel importante actuando sinérgicamente junto a la quimiorecepción por contacto, tal y como se ha sugerido en otros cerambícidos (Fukaya et al., 1999; Lopes et al., 2005). 


\section{Conclusiones}

El estudio evidencia la presencia de feromona sexual de contacto en hembras de C. welensii, reconocida mediante quimiorecepción (palpos bucales) por machos e incitadora de la cópula. Los compuestos candidatos a participar en la actividad feromonal de contacto podrían localizarse tanto en élitros como en protórax, pudiendo actuar como componentes mayoritarios $11-\mathrm{Me}-\mathrm{C} 29, \mathrm{C} 27,2-\mathrm{Me}-\mathrm{C} 26$ y $11 / 13-\mathrm{Me}-$ C27, y como componentes minoritarios 11/12/13-Me-C26 y C28. La distinción sexual en $C$. welensii podría deberse a la diferente proporción en que algunos componentes cuticulares estén presentes en machos y hembras, y no a la existencia de compuestos claramente diferenciadores entre sexos. Cerambyx welensii sigue el comportamiento de preparación a la cópula encontrado en otros cerambícidos (contacto antenal, palpado y arqueo abdominal), resultando la retirada de compuestos cuticulares de las hembras inhibidora de la respuesta sexual de los machos, y la restitución de los extractos cuticulares incitadora de la respuesta sexual.

\section{Bibliografía}

Allison, J.D.; Borden, J.H.; Seybold, J.H.; 2004. A review of the chemical ecology of the Cerambycidae (Coleoptera). Chemoecology 14: 123-150. https://doi.org/10.1007/s0 0049004-0277-1

Barbour, J.; Lacey, E.; Hanks, L.; 2007. Cuticular hydrocarbons mediate mate recognition in a species of longhorned beetle (Coleoptera: Cerambycidae) of the primitive subfamily Prioninae. Ann. Entomol. Soc. Am. 100: 333-338. https://doi.org/10.1603/00138746(2007)100[333:CHMMRI]2.0.CO;2

Buse, J.; Schröderb, B.; Assmann, T.; 2007. Modelling habitat and spatial distribution of an endangered longhorn beetle - A case study for saproxylic insect conservation. Biol. Conserv. 137: 372-381. https://doi.org/10.1016/j.biocon.2007.02.025

Carrasco, A.; y cols., 2009. Procesos de Decaimiento Forestal (la Seca), Situación del Conocimiento. Consejería de Medio Ambiente, Junta de Andalucía, 112 pp. Córdoba.

Chiang, Y.N.; Tan, K.J.; Chung, H.; Lavrynenko, O.; Shevchenko, A.; Yew, J.Y.; 2016. Steroid hormone signaling is essential for pheromone production and oenocyte survival. PLoS Genet. 12: e1006126. https://doi.org/10.1371/journal.pgen.1006126

de la Cruz, J.; 2006. Entomología, Morfología y Fisiología de los Insectos (pp. 33-126). Palmira: Editorial Universidad Nacional de Colombia.

del Moral, J.; 1994. Cerambyx spp.: historia de una plaga de las dehesas extremeñas, PhytomaEspaña, 60: 18-24.

Duque-Lazo, J.; Navarro-Cerrillo, R.; 2017. What to save, the host or the pest? The spatial distribution of xylophage insects within the Mediterranean oak woodlands of Southwestern Spain. For. Ecol. Manag 92: 90-104. https://doi.org/10.1016/j.foreco. 2017.02.047

Fukaya, M.; Akino, T.; Yasuda, T.; Tatsuki, S.; Wakamura, S.; 1999. Mating sequence and evidence for synergistic component in female contact sex pheromone of the white-spotted longicorn beetle, Anoplophora malasiaca (Thomson) (Coleoptera: Cerambycidae). Entomol. Sci. 2: 183-187. 
Ginzel, M.; 2010. Hydrocarbons as contact pheromones of longhorned beetles (Coleoptera: Cerambycidae). In: Blomquist, G.; Bagnères, A.; (eds.), Insect Hydrocarbons: Biology, Biochemistry, and Chemical Ecology (pp. 375-389). Cambridge: Cambridge University Press. https://doi.org/10.1017/CBO9780511711909.018

Ginzel, M.D.; Blomquist, G.J.; Millar, J.G.; 2003a. Role of contact pheromones in mate recognition in Xylotrechus colonus. J. Chem. Ecol. 29: 533-545.

Ginzel, M.D.; Millar, J.G.; Hanks, L.M.; 2003b. (Z)-9-Pentacosene - contact sex pheromone of the locust borer, Megacyllene robiniae. Chemoecol. 13:135-141. https://doi.org /10.1007/s00049-003-0239-z

Ginzel, M.D.; Blomquist, G.; 2016. Insect Hydrocarbons: Biochemistry and Chemical Ecology. In: Cohen, E.; Moussian, B.; (eds.), Extracellular composite matrices in arthropods. Switzerland: Springer International Publishing. pp. 221-252. https://doi.org/ 10.1007/978-3319-40740-1_7

Ibeas, F.; Gemeno, C.; Díez, J.J.; Pajares, J.A.; 2008. Female recognition and sexual dimorphism of cuticular hydrocarbons in Monochamus galloprovincialis (Coleoptera: Cerambycidae). Ann. Entomol. Soc. Am. 102: 317-325. https://doi.org/10.1603/008.10 2.0214

Lacey, E.; Ginzel, M.; Millar, J.; Hanks, L.; 2008. 7_methylheptacosane is a major component of the contact sex pheromone of the cerambycid beetle Neoclytus acuminatus acuminatus. Physiol. Entomol. 33: 209-2016. https://doi.org/10.1111/j.1365-3032. 2008.00624.x

Lockey, K.; 1985. Insect cuticular lipids. Comp. Biochem. Physiol. B 81: 263-267. https:// doi.org/10.1016/0305-0491(85)90311-6

Lopes, O.; Marques, P.; Araújo, J.; 2005. The role of antennae in mate recognition in Phoracantha semipunctata (Coleoptera: Cerambycidae). J. Insect Behav. 18: 243-257. https://doi.org/10.1007/s10905-005-0478-7

López-Pantoja, G.; Domínguez, L.; Sánchez-Osorio, I.; 2008. Mark-recapture estimates of the survival and recapture rates of Cerambyx welensii Küster (Coleoptera Cerambycidae) in a cork oak dehesa in Huelva (Spain). Cent. Eur. J. Biol. 3: 431-441. https:// doi.org/10.2478/s11535-008-0044-3

Martín, J.; Cabezas, J.; Buyolo, T.; Patón, D.; 2005. The relationship between Cerambyx spp. damage and subsequent Biscogniauxia mediterranum infection on Quercus suber forests. For. Ecol. Manag. 216: 166-174. https://doi.org/10.1016/j.foreco.2005.05.027

Navarro, R.M.; Fernández, P.; 2001. Evaluation de dégats produits par le déperisement du chêneliege en Andalusie. Selviculture of cedar (Cedrus atlantica End. M.) and cork oak (Quercus suber L.). IUFRO.

Pajares, J.A.; Álvarez, G.; Ibeas, F.; Gallego, D.; Hall, D.R.; Farman, D.I.; 2010. Identification and field activity of male-produced aggregation pheromone in the pine sawyer beetle, $\mathrm{Mo}$ nochamus galloprovincialis. J. Chem. Ecol. 36: 570-583. https://doi.org/ 10.1007/s10886010-9791-5

Rodríguez, A.; 2014. Mejora en las estrategias de control de cerambícidos en el cultivo de la vid. Universidad de León. 338 pp

Sacristán, A.; Sánchez-Husillos, E.; Hall, DR.; Pajares, J.A.; 2017. Feromonas de contacto implicadas en el reconocimiento sexual en Monochamus galloprovincialis y Monochamus sutor (Coleoptera: Cerambycidae). Panel presentado en el $7^{\circ}$ Congreso Forestal Español, Plasencia.

Sallé, A.; Nageleisen, L.M.; Lieutier, F.; 2014. Bark and wood boring insects involved in oak declines in Europe: current knowledge and future prospects in a context of climate change. For. Ecol. Manag. 328: 79-93. https://doi.org/10.1016/j.foreco.2014.05. 027 
Sánchez-Osorio, I.; Tapias, R.; López, G.; Domínguez, L.; 2012. Pautas de apareamiento y localización intraespecífica en Cerambyx welensii Küster (Coleoptera: Cerambycidae). Cuad. Soc. Esp. Cienc. For. 36: 119-125.

Sánchez-Osorio, I.; López-Pantoja, G.; Paramio, A.M.; Lencina, J.L.; Gallego, D.; Domínguez, L.; 2016. Field attraction of Cerambyx welensii to fermentation odors and host monoterpenes. J. Pest. Sci. 89: 59-68. https://doi.org/10.1007/s10340-015-0654-2

Sánchez-Osorio, I.; López-Pantoja, G.; Tapias, R.; Pareja-Sánchez, E.; Domínguez, L.; 2019. Monoterpene emission of Quercus suber L. highly infested by Cerambyx welensii Küster. Ann. For. Sci. 89. https://doi.org/10.1007/s13595-019-0879-y

Silk, P.J.; Sweeney, J.; Wu, J.; Sopow, S.; Mayo, P.D.; Magee, D.; 2011. Contact sex pheromones identified for two species of longhorned beetles (Coleoptera: Cerambycidae) Tetropium fuscum and T. cinnamopterum in the Subfamily Spondylidinae. Env. Entomol. 40: 714-726. https://doi.org/10.1603/EN10213

Soria, F.J.; Villagrán, M.; Ocete, M.; 1994. Estudios prospectivos de los principales perforadores del alcornoque en la Sierra Norte de Sevilla. Bol. San.Veg. Plagas 20: 643 -651.

Tiberi, R.; Branco, M.; Bracalini, M.; Croci, F.; Panzavolta, T.; 2016. Cork oak pests: a review of insect damage and management. Ann. For. Sci. https://doi.org/10.1007/s1359 5-0150534-1

Torres-Vila, L.M.; Sanchez-González, A.; Ponce-Escudero, F.; Martín-Vertedor, D.; FerreroGarcía, J.J.; 2012. Assessing mass trapping efficiency and population density of Cerambyx welensii Küster by mark-recapture in dehesa open woodlands. Eur. J. Forest. Res. 131: 1103-1116. https://doi.org/10.1007/s10342-011-0579-0

Wang, Q.; 2002. Sexual selection of Zorion guttigerum Westwood (Coleoptera: Cerambycidae) in relation to body size and color. J. Insect Behav. 15: 701-713.

Wang, Q.; Zeng, W.; Chen, L.; Li, J.; Yin, X.; 2002. Circadian reproductive rhytms, pair bonding, and evidence for sex specific pheromones in Nadezhdiella cantori, J. Insect Behav. 15: 527-539. https://doi.org/10.1023/A:1016333317564

WWW/Adena, 2006. La dehesa en los Programas de Desarrollo Rural 2007-13. Propuesta. $34 \mathrm{pp}$. 